\title{
SUBGROUPS OF SEQUENCES AND PATHS
}

\section{B. JAMISON ${ }^{1}$ AND S. OREY ${ }^{2}$}

0 . It is well known and easy to prove that if a measurable subgroup $G$ of $n$-dimensional Euclidean space $R^{n}$ has positive $n$-dimensional Lebesgue measure, then $G=R^{n}$. In the first section we give an analogue of this result for subgroups of real-valued sequences, where the space $R^{\infty}$ of all such sequences is given product normalized Gaussian measure. In the second section we use this result to sharpen and extend a zero-one law of Cameron and Graves [1].

1. In this section, $R$ is the set of real numbers, $R^{n}$ is $n$-dimensional Euclidean space, and $R^{\infty}$ is the set of all sequences $\left(x_{1}, x_{2}, \cdots\right)$ of real numbers. $B$ is the class of Borel subsets of $R$, and $B^{n}\left(B^{\infty}\right)$ is the corresponding product $\sigma$-field over $R^{n}\left(R^{\infty}\right)$. We will use $\mu$ to denote the Gaussian measure on $B^{\infty}$ mentioned above. We say that a subset of $R^{\infty}$ is measurable if it is in the completion of $B^{\infty}$ with respect to $\mu$.

THEOREM. If $G$ is a measurable subgroup of $R^{\infty}$, then either $\mu(G)=0$ or $\mu(G)=1$.

Lemma. Suppose that $f$ and $g$ are bounded functions on $R^{\infty}$, each measurable with respect to the $\mu$-completion of $B^{\infty}$. For each $x \in R^{n}$, let $g_{x}$ be the function on $R^{\infty}$ defined by

$$
g_{x}(y) f\left(\left(x_{1}, \cdots, x_{n}, 0, \cdots\right)+y\right) .
$$

Let $h$ be defined on $R^{n}$ by

$$
h(x)=\int f(y) g_{x}(y) \mu(d y) .
$$

Then $h$ is measurable with respect to $B^{n}$. If $f$ is continuous with respect to the product topology on $R^{\infty}, h$ is continuous on $R^{n}$.

Proof. $\mu$ is a product measure each of whose factors has as density the function $\phi$, where

$$
\phi(t)=(2 \pi)^{-1 / 2} \exp \left(-t^{2} / 2\right), \quad t \in R .
$$

For each $x \in R^{\infty}$, let

Received by the editors February 18, 1969.

1 Partial support by AFOSR.

2 Partial support by NSF. 


$$
\begin{array}{r}
A(x, z)=\int_{R^{n}} f\left(y_{1}, \cdots, y_{n}, z_{1}, \cdots\right) g\left(x_{1}+y_{1}, \cdots, x_{n}+y_{n}, z_{1}, \cdots\right) \\
\cdot \phi\left(y_{1}\right) \cdots \phi\left(y_{n}\right) d y_{1} \cdots d y_{n} .
\end{array}
$$

It follows from Fubini's theorem that

$$
h(x)=\int A(x, z) \mu(d z) .
$$

The translation-invariance of Lebesgue measure on $R^{n}$ yields

$$
\begin{array}{r}
A(x, z)=\int f\left(y_{1}-x_{1}, \cdots, y_{n}-x_{n}, z_{1}, \cdots\right) g\left(y_{1}, \cdots, y_{n}, z_{1}, \cdots\right) \\
\cdot \phi\left(y_{1}-x_{1}\right) \cdots \phi\left(y_{n}-x_{n}\right) d y_{1} \cdots d y_{n} .
\end{array}
$$

Fix $z$. If $f$ is continuous, the integrand is a continuous function of $x \in R^{n}$, and then so is $A(x, z)$ by the bounded convergence theorem. Another application of the bounded convergence theorem shows that $h(x)=\int A(x, z) \mu(d z)$ is also continuous. Suppose $f$ is bounded and measurable with respect to the $\mu$-completion of $B^{\infty}$. Then $f$ is the bounded $\mu$-almost everywhere limit of a sequence $\left\{f_{n}\right\}$ of functions continuous on $R^{\infty}$, and for each $x \in R^{n}$

$$
h(x)=\lim _{n} \int f_{n}(y) g_{x}(y) \mu(d y),
$$

again by the bounded convergence theorem. But the pointwise limit of functions continuous on $R^{n}$ is Borel measurable on $R^{n}$, and the proof is complete.

If we take $f \equiv 1$ we have the following

Corollary. If $f$ is bounded and measurable with respect to the $\mu$ completion of $B^{\infty}$, then $\int f_{x} d \mu$ is continuous on $R^{n}$.

It should be noted that the map $x \rightarrow \mu(A+x)$ is not in general continuous on $R^{\infty}$. In fact, let

$$
A=\left\{x \in R^{\infty}: \lim (1 / n)\left(x_{1}+\cdots+x_{n}\right)=0\right\} .
$$

Then $\mu(A)=1$ by virtue of the strong law of large numbers. However, if $x^{(k)}=(1 / k, 1 / k, \cdots), \mu\left(x^{(k)}+A\right)=0$, even though $x^{(k)} \rightarrow 0$ in the product topology on $R^{\infty}$.

Proof of THEOREM. If $x \in R^{\infty}$ and $S \subset R^{\infty}$, then $x+S$ is the set of all sequences $x+y$ where $y \in S$, the addition being performed componentwise. We say that $x \in R^{\infty}$ is $n$-dimensional if $x_{m}=0$ for all $m>n$. Let 
$G^{*}$ be the union of all cosets $x+G$ as $x$ ranges over all $n$-dimensional sequences and $n$ ranges over the positive integers. Clearly $G^{*}$ has the property that if $x \in G^{*}$ and if $y$ differs from $x$ in only finitely many terms then $y \in G^{*}$. Once we show that $G^{*}$ is measurable it follows that $G^{*}$ is a tail event, so either $\mu\left(G^{*}\right)=0$ or $\mu\left(G^{*}\right)=1$ [4, p. 229]. Consider the map $\gamma: x \rightarrow \mu(x+G)$ of $R^{\infty}$ in to $[0,1]$. For fixed $n$, the restriction of $\gamma$ to the set of $n$-dimensional sequences $x$ induces a map $\gamma^{(n)}$ of $R^{n}$ into $[0,1]$ which is continuous, by virtue of the above corollary. But cosets $x+G$ and $y+G$ are either disjoint or identical, so the range of $\gamma^{(n)}$ is countable. Since $R^{n}$ is connected, this is possible only if $\gamma^{(n)}$ is constant, the constant necessarily equal to $\gamma^{(n)}(0,0, \cdots)=\mu(G)$. If we assume that $\mu(G)>0$, it follows that $\{x+G: x$ finite dimensional $\}$ is a finite class of sets. Since each of its members is a measurable subset of $R^{\infty}$, so is its union $G^{*}$. Therefore $G^{*}$ is a tail set. Since $G^{*} \supset G$, we have $\mu\left(G^{*}\right)=1$.

We complete the proof by showing that $G^{*}=G$. From what we have already shown it follows that there are integers $m$ and $n$ and $n$-tuples $(x(i, 1), \cdots, x(i, n)), 1 \leqq i \leqq m$ such that

$$
G^{*}=G \cup\left(y_{1}+G\right) \cup \cdots \cup\left(y_{m}+G\right),
$$

where

$$
y_{i}=(x(i, 1), \cdots, x(i, n), 0,0, \cdots), \quad 1 \leqq i \leqq m .
$$

Let

$$
A_{0}=\left\{\left(x_{1}, \cdots, x_{n}\right):\left(x_{1}, \cdots, x_{n}, 0, \cdots\right)+G=G\right\},
$$

and for each $i=1, \cdots, m$, let

$$
A_{i}=(x(i, 1), \cdots, x(i, n))+A_{0},
$$

the addition being performed in $R^{n}$. If we observe that, because of the lemma,

$$
\left(x_{1}, \cdots, x_{n}\right) \rightarrow \mu\left(\left(\left(x_{1}, \cdots, x_{n}, 0, \cdots\right)+G\right) \cap G\right)
$$

is Borel measurable, we see that $A_{0}$ is Borel measurable, as are, therefore, its translates $A_{i}, 1 \leqq i \leqq m$. Since any coset of the form $\left(x_{1}, \cdots, x_{n}, 0, \cdots\right)+G$ has to be either $G$ (in which case $\left(x_{1}, \cdots, x_{n}\right)$ $\left.\in A_{0}\right)$ or $y_{i}+G$ for some $i=1, \cdots, m$ (in which case $\left(x_{1}, \cdots, x_{n}\right) \in A_{i}$ ), it follows that $\left\{A_{0}, \cdots, A_{m}\right\}$ is a partition of $R^{n}$ into Borel measurable sets. One of them, say $A_{k}$, has to have positive $n$-dimensional Lebesgue measure, in which case, as is well known, its difference set $A_{k}-A_{k}$ has to contain a cube. But $A_{k}-A_{k}=A_{0}$, and $A_{0}$ is a subgroup 
of $R^{n}$, so it follows that $A_{0}=R^{n}$, whence $G^{*}=G$, and the proof is complete.

2. In this section $\Omega=C[0,1]$, the class of all continuous real valued functions. For each $0 \leqq t \leqq 1$, the function $X_{t}$ is defined by

$$
X_{t}(\omega)=\omega(t), \quad \omega \in \Omega .
$$

We define $\mathcal{F}$ to be the smallest $\sigma$-field over $\Omega$ relative to which each of the functions $X_{t}$ is measurable. If $P$ is a probability measure of $F$, the quadruple $\left(\Omega, \mathcal{F}, P,\left\{X_{t}\right\}_{0 \leqq t \leqq 1}\right)$ is called a process. The members of $\Omega$ are referred to as paths; we are considering, of course, only processes on $[0,1]$ all of whose paths are continuous. In fact, we further restrict ourselves only to Gaussian processes with mean 0 , that is, those for which

$$
P\left\{\left(X_{t_{1}}, \cdots, X_{t_{n}}\right) \in B\right\}, \quad B \in \mathbb{B}^{n},
$$

defines a Gaussian measure on $R^{n}$ for each subdivision $0 \leqq t_{1}<\ldots$ $<t_{n} \leqq 1$ of $[0,1]$ and each $n=1,2, \cdots$ (see $[4$, p. 462], where "strongly normal" is used for "Gaussian") and where $E\left(X_{t}\right)=0$, $0 \leqq t \leqq 1$. " $E$ " denotes integration over $\Omega$ with respect to the measure $P$.) Such processes are completely determined by their covariance function

$$
\Gamma(s, t)=E\{X(s) X(t)\}, \quad s, t \in[0,1] .
$$

Mercer's Theorem states that the expansion

$$
\Gamma(s, t)=\sum_{n=0}^{\infty} \lambda_{n} \Psi_{n}(s) \Psi_{n}(t)
$$

converges uniformly on $[0,1] \times[0,1]$, where the $\lambda_{n}$ 's and $\Psi_{n}$ 's are, respectively, the positive eigenvalues and eigenfunctions of the integral equation with kernel $\Gamma(s, t)$ :

$$
\int_{0}^{1} \Gamma(s, t) \Psi_{n}(t) d t=\lambda_{n} \Psi_{n}(s), \quad 0 \leqq s \leqq 1 .
$$

We define

$$
\xi_{n}(\omega)=\frac{1}{\sqrt{ } \lambda_{n}} \int_{0}^{1} X_{t}(\omega) \Psi_{n}(t) d t, \quad \omega \in \Omega
$$

The $\xi_{n}(\omega)$ 's thus obtained form a family of independent random variables on $(\Omega, F, P)$, each normal with mean 0 and variance 1 . If we define $X_{n}, n=0,1, \ldots$ by 


$$
X_{n}(t, \omega)=\sum_{k=1}^{n} \sqrt{ } \lambda_{n} \xi_{n}(\omega) \Psi_{n}(t),
$$

then Mercer's Theorem shows that $E\left|X_{n}(t)-X_{t}\right|^{2} \rightarrow 0$ uniformly in $t$ [4, pp. 478-479]. The expression

$$
X(t, \omega)=\sum \sqrt{ } \lambda_{n} \xi_{n}(\omega) \Psi_{n}(t), \quad \omega \in \Omega, \quad 0 \leqq t \leqq 1
$$

is known as the Karhunen-Loève expansion of the process $\left\{X_{t}, 0 \leqq t \leqq 1\right\}$. This expansion is valid for any real-valued Gaussian process on $[0,1]$ with $E\left(X_{t}\right) \equiv 0$. Since the functions $\Psi_{n}$ are continuous on $[0,1]$, a sufficient condition for the continuity of almost all paths is that convergence of the right hand side of (3) is uniform in $t$ for almost all $\omega$. This condition of almost sure uniformity is also necessary for the continuity of the paths [2] so it prevails for the processes under consideration here.

We say that a subset of $\Omega$ is measurable if it is in the completion of $\mathcal{F}$ with respect to $P$. Here is an application of Theorem 1 to continuous Gaussian processes.

Theorem 2. Let $\left(\Omega, \mathcal{F}, P,\left\{X_{t}, 0 \leqq t \leqq 1\right\}\right)$ be a Gaussian process with continuous sample paths and with $E\left(X_{t}\right) \equiv 0$. If $G$ is a measurable subgroup of $\Omega$, then either $P(G)=0$ or $P(G)=1$.

Proof. Referring to (2), define $\kappa$ on $\Omega$ into $R^{\infty}$ by

$$
\kappa(\omega)=\left(\xi_{1}(\omega), \xi_{2}(\omega), \cdots, \xi_{n}(\omega), \cdots\right) .
$$

The definition of the $\xi_{n}$ 's shows that $\kappa$ is $\mathcal{F}-B$ measurable. The Karhunen-Loève expansion shows that $\kappa$ is measure preserving in the sense that $P \circ \kappa^{-1}=\mu$, so the measurability holds even if $F$ and $B^{\infty}$ are replaced by their completions under $P$ and $\mu$ respectively. The proof should now proceed in the obvious way: $\kappa$ is linear, so $G^{\prime}=\kappa(G)$ is a subgroup of $R^{\infty}$ to which Theorem 1 applies, and Theorem 2 follows. However, $\kappa(G)$ is a direct image under a measurable map, not an inverse image, so its measurability is in question. To get around this difficulty, we complicate the argument as follows. Let $H$ be the set of $\omega$ 's in $\Omega$ for which the functions $X_{n}(\omega, t)$ converge to $X_{t}(\omega)$ uniformly in $t$. Since $X_{n}(\omega, t)$ is $\mathfrak{F}$-measurable for each fixed $t$, and since a sequence of continuous functions converges uniformly on $[0,1]$ if and only if it converges uniformly on, say, the binary rationals in $[0,1]$, it follows in a rather straightforward way that $H \in \mathcal{F}$. But $H$ is a group, and, by Theorem 3.1 of [2], $P(H)=1$. Furthermore, $H^{\prime}=\kappa(H)$ is measurable, since $\left(x_{1}, x_{2}, \cdots\right) \in H^{\prime}$ if and only if the functions $\sum_{k=1}^{n} \sqrt{ } \lambda_{k} x_{k} \Psi_{k}(t)$ converge uniformly on $[0,1]$ as $n \rightarrow \infty$. 
In fact it is easy to see that $\kappa$ induces a one-to-one bimeasurable and measure-preserving correspondence between $H$ and $H^{\prime}$, the bimeasurability holding even if $\mathcal{F}$ and $B^{\infty}$ are enlarged to their respective completions. Now suppose that $P(G)>0$. Let $G_{1}=G \cap H ; G_{1}$ is also a group, and $P\left(G_{1}\right)=P(G)$. Also because of bimeasurability $G_{2}=\kappa(G \cap H)$ is measurable. Thus $G_{2}$ is a measurable subgroup of $R^{\infty}$, and Theorem 1 applies. Since $\mu\left(G_{2}\right)=P\left(G_{1}\right)=P(G)>0, \mu\left(G_{2}\right)=1$, so $P(G)=1$. The proof is complete.

Cameron and Graves (Theorem 3 of [1]) have proved this result in the case where $\left\{X_{t}, 0 \leqq t \leqq 1\right\}$ is the Wiener process and where $G$, in addition to being a group, is closed under scalar multiplication by rationals. Examples of subgroups $G$ of $C[0,1]$ to which Theorem 2 is applicable are given by (1) the set of all $f \in C[0,1]$ for which $\int_{0}^{1} \Psi(t) f(t) d t<\infty$, where $\Psi$ is a fixed measurable but unbounded function on $[0,1]$, or an arbitrary member of some class of such functions, (2) the set of all members of $C[0,1]$ satisfying a Lipschitz or Hölder condition.

Using completely different techniques, Kallianpur [3] has shown that $P(G)$ is 0 or 1 even if the Gaussian process has discontinuous paths, provided $G$ is a measurable $r$-module, that is, a group closed under multiplication by rationals. It would be interesting to exhibit a case for which $G$ is a group, $P(G)=1$, but $G$ is not a module. Kallianpur also shows that if $G$ is a group belonging not only to the $\mu$-completion of $B^{\infty}$ but to $B^{\infty}$ itself, then $P(G)=0$ or $P(G)=1$. Again, this result does not require the path continuity of the process. His results render almost inescapable the conjecture that the condition of sample path continuity can be dropped from our Theorem 2 .

\section{REFERENCES}

1. R. H. Cameron and R. E. Graves, Additive functionals on a space of continuous functions. I, Trans. Amer. Math. Soc. 70 (1951), 160-176. MR 12, 718.

2. A. M. Garsia, E. Rodemich and H. Rumsey, Jr., A real variable lemma and the continuity of paths of some Gaussian processes (mimeographed).

3. G. Kallianpur, Zero-one laws for Gaussian processes (preprint).

4. M. Loève, Probability theory, 3rd ed., Van Nostrand, Princeton, N. J., 1963. MR 34 \#3596.

University of California, San Diego 